\title{
Fibrodisplasia Óssea Craniofacial: Apresentação de 2 Casos e Revisão da Literatura
}

Craniofacial Fibrous Dysplasia: Presentation of 2 Cases and Literature Review

Diana Pinto Silva ${ }^{1}$, António Lima ${ }^{1}$, Nuno Costa² ${ }^{2}$ Sérgio Vilarinho ${ }^{3}$, Afonso Almeida Pinto ${ }^{4}$, Luís Dias ${ }^{5}$

\section{RESUMO}

A fibrodisplasia óssea é uma patologia congénita rara com predileção pelos ossos craniofaciais, envolvendo frequentemente os seios perinasais, órbita e base do crânio.

Os autores apresentam 2 casos de fibrodisplasia óssea monostótica craniofacial e realizam uma breve revisão da literatura sobre o tema. O primeiro trata-se de um caso de fibrodisplasia óssea do frontal, num homem de 20 anos, e o segundo de um caso de fibrodisplasia óssea do esfenoide, num homem de 65 anos. O primeiro caso, dada a idade jovem, crescimento e repercussão estética da lesão, foi submetido a cirurgia, não evidenciando sinais de recidiva após um ano de seguimento. O segundo caso, tratou-se de um achado imagiológico sem sinais de crescimento, num homem mais velho e assintomático, pelo que se optou pelo tratamento conservador e vigilância. Ambos foram orientados de forma multidisciplinar e ilustram a diversidade de apresentação, orientação e evolução que a mesma patologia pode assumir.

PALAVRAS-CHAVE: Displasia Fibrosa, Fibrodisplasia Monostótica; Fibrodisplasia Óssea; Fibrodisplasia Poliostótica; Ossos Faciais 


\section{ABSTRACT}

A Craniofacial fibrous dysplasia is a rare congenital pathology of the bone, with predilection for craniofacial bones, frequently involving the nasal sinuses, orbit and skull base.

The authors present 2 cases of craniofacial monostotic fibrous dysplasia and perform a brief revision of literature about this matter. The first case is a frontal fibrous dysplasia in a 20-year-old man, and the second case is about sphenoid fibrous dysplasia in a 65-year-old man. The first case, given the young age, growth and aesthetic repercussion, was submitted to surgery, without signs of relapse after one year of follow-up. The second one was an accidental finding in an older and asymptomatic man, reason why he underwent conservative treatment and observation.

Both were multidisciplinary managed at our institution and show the variability of presentation, orientation and evolution of the same pathology.

KEYWORDS: Facial Bones; Fibrous Dysplasia of Bone; Fibrous Dysplasia, Monostotic; Fibrous Dysplasia, Polyostotic

\section{INTRODUÇÃO}

A fibrodisplasia óssea (FO) é uma patologia congénita rara, caracterizada pela substituição do osso esponjoso por tecido fibrótico. Ocorre devido à ativação de mutações somáticas na subunidade $\alpha$ do gene GNAS, o que resulta na inibição da proliferação e diferenciação das células ósseas estromais, levando à substituição do osso normal por tecido fibroso e reticulado. ${ }^{1,2}$

Apresenta-se sob duas formas, FO monostótica (FOM) quando envolve apenas um osso ou FO poliostótica (FOP) quando há envolvimento de vários ossos. A FOM é cerca de quatro vezes mais prevalente que a FOP, sendo as localizações mais comuns os ossos craniofaciais, seguidos do fémur proximal e das costelas. ${ }^{3}$ Ocorre muitas vezes associada à síndrome de McCune Albright (SMA) caracterizada pela tríade de FOP, manchas cutâneas do tipo "café com leite" e endocrinopatia, como puberdade precoce. Quando a FO se associa à SMA, acomete os ossos craniofaciais em $90 \%$ e a base do crânio em mais de 95\% dos casos, o que evidencia a necessidade de pesquisa desta associação, sobretudo quando ocorre nestas localizações. ${ }^{4}$

De seguida, os autores apresentam dois casos de FOM craniofacial dos seios perinasais frontal e esfenoide, respetivamente, realizando associadamente uma revisão da literatura sobre este tema.

\section{CASO 1}

Homem de 20 anos com tumefação frontal direita de crescimento progressivo com um ano de evolução. Sem outras queixas associadas ou antecedentes de relevo. No exame objetivo apresentava volumosa tumefação na região frontal, supraciliar direita e glabela, dura, imóvel, sem sinais inflamatórios. Não apresentava alterações na endoscopia nasal bem como no restante exame de
Otorrinolaringologia (ORL). Sem evidência de lesões cutâneas associadas (Fig. 1). Como estudo complementar foram realizadas tomografia computorizada (TC) e ressonância magnética (RM). A TC mostrou uma lesão expansiva centrada à estrutura óssea fronto-etmoidal direita, com áreas fibróticas e de calcificação, extensão naso-etmoidal anterior, erosão da tábua interna do seio frontal e expressão epidural. A RM destacou a estrutura heterogénea da lesão, com áreas pseudo-quísticas, de fibrose e calcificação, com sinal pouco intenso em T1 e hiperintensas em T2 (Fig. 2). O doente foi orientado para intervenção multidisciplinar com equipa de ORL e Neurocirurgia, realizando-se exérese de lesão fibrodisplásica frontal e da fossa anterior através de craniotomia bicoronal com encerramento da dura-máter e do seio frontal com retalho de pericrânio e reconstrução do defeito ósseo por cranioplastia (Fig. 3).

A histologia revelou FOM frontal do subtipo cístico. A TC pós-operatória mostrou exérese radical da lesão. $O$ doente teve alta no quarto dia pós-operatório, sem défices neurológicos, com bom resultado estético. Encontra-se em seguimento há um ano, sem evidência clínica ou imagiológica de recidiva (Fig. 4).

\section{CASO 2}

Homem de 65 anos, referenciado a ORL por hipoacusia e acufeno com vários anos de evolução, não apresentando outras queixas ORL, oftalmológicas ou sistémicas. No exame objetivo não apresentava alterações na otoscopia, e na realização da endoscopia nasal foi verificada presença de uma tumefação na nasofaringe que condicionava apagamento da fosseta de Rosenmuller à esquerda. Estes achados motivaram a realização de uma TC da faringe que revelou alterações compatíveis com FOM esfenoidal à esquerda, condicionando obliteração 


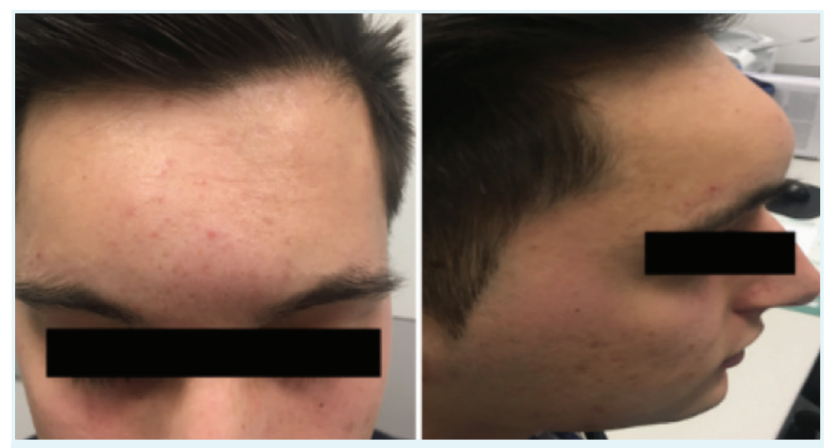

FIGURA 1. Tumefação volumosa na região frontal, supra-ciliar direita e glabella, sem sinais inflamatórios ou alterações cutâneas associadas.
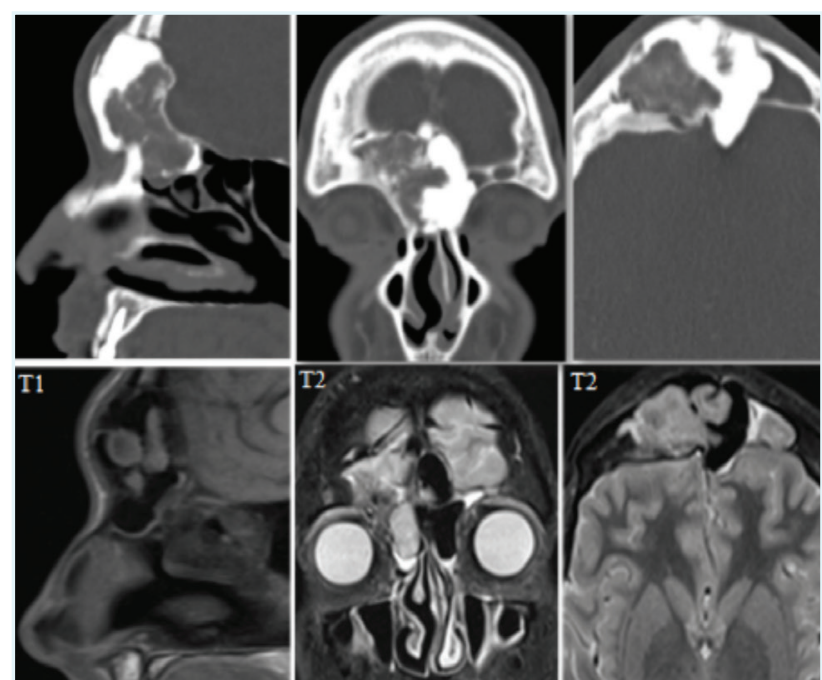

FIGURA 2. A TC mostrou lesão expansiva centrada à estrutura óssea fronto-etmoidal, com áreas fibróticas e de calcificação, extensão naso-etmoidal anterior, erosão da tábua interna do seio frontal e expressão epidural. A RM destacou a estrutura heterogénea, com áreas pseudo-quísticas, de fibrose e calcificação com sinal pouco intenso em T1 e hipercaptantes em T2.

praticamente total do seio esfenoidal, envolvimento do corpo, grande asa e apófise pterigoide ipsilateral (Fig. 5). A RM revelou ausência de envolvimento intracraniano ou de invasão de tecidos moles adjacentes. Procedeu-se a discussão multidisciplinar do caso entre ORL, Neurocirurgia, Oftalmologia e Endocrinologia, tendo sido decidido tratamento conservador com bifosfonatos para controlo do crescimento da lesão, associado a observação, vigilância clínica, oftalmológica e imagiológica periódicas. $\bigcirc$ doente encontra-se em seguimento há 2 anos, mantendo-se assintomático, sem alterações da acuidade visual, e sem evidência clínica e imagiológica de progressão da doença.

\section{DISCUSSÃO}

A FO é uma condição rara do processo de modelagem óssea, representando 5\% dos tumores ósseos benignos. ${ }^{1}$ As manifestações clínicas dependem da forma e localização da doença. Pode ocorrer nos seios perinasais, sendo mais frequente no esfenoide, seguido do etmoide, maxilar e menos comum no frontal, não se acompanhando da sintomatologia típica da rinossinusite. ${ }^{5}$ Pode ocorrer envolvimento da órbita, nervo ótico, base do crânio e compressão do parênquima encefálico, o que enfatiza a necessidade da discussão e abordagem multidisciplinar adequadas. $^{5}$

Tipicamente manifesta-se na infância e adolescência com deformidade óssea de crescimento indolente. A progressão natural é geralmente insidiosa, sobretudo nos adultos. Nos pacientes em fase de crescimento pode ter uma evolução mais rápida devido à elevada atividade osteoblástica, formação de aneurismas císticos do osso ou mucocelos, levando a deformação estética evidente e compressão de estruturas adjacentes, assistindo-se à estabilização no término do crescimento.1,3 A transformação sarcomatosa maligna ocorre em menos de 1\% dos casos.

A investigação imagiológica geralmente é realizada por TC e RM, sendo as imagens bastante características e essenciais para avaliação da localização, extensão e caracterização da fase de atividade da doença.

Os principais diagnósticos diferenciais, definidos sobretudo por critérios radiológicos, são o fibroma ossificante, o osteoma, o osteocondroma, o meningioma da base do crânio e o quisto ósseo aneurismático. O fibroma ossificante é o principal diagnóstico diferencial, sobretudo da forma monostótica, dada a semelhança da apresentação clínica e radiológica. $\bigcirc$ comportamento geralmente mais agressivo e melhor definição de margem nos exames imagiológicos são características do fibroma ossificante, sendo o diagnóstico definitivo obtido com a anatomopatologia.

A abordagem multidisciplinar é crucial, e sobretudo na forma craniofacial, e deverá incluir a Otorrinolaringologia ou Cirurgia Máxilo-Facial, a Neurocirurgia, a Oftalmologia e a Endocrinologia.

O tratamento depende da forma, estado de atividade da doença, idade e fase de crescimento no diagnóstico, bem como do envolvimento de estruturas adjacentes.

O tratamento cirúrgico está indicado nos casos de deformidade estética e rápido crescimento com compressão de estruturas, realizando-se idealmente na fase de estabilização do crescimento e da forma ativa da doença. ${ }^{3,6}$

A literatura relativamente ao tratamento cirúrgico da FO dos seios perinasais é escassa. As guidelines de abordagem da FO craniofacial proposta por Lee e colaboradores, definem que o tratamento cirúrgico da FO dos seios perinasais pode ser realizado através de cirurgia 

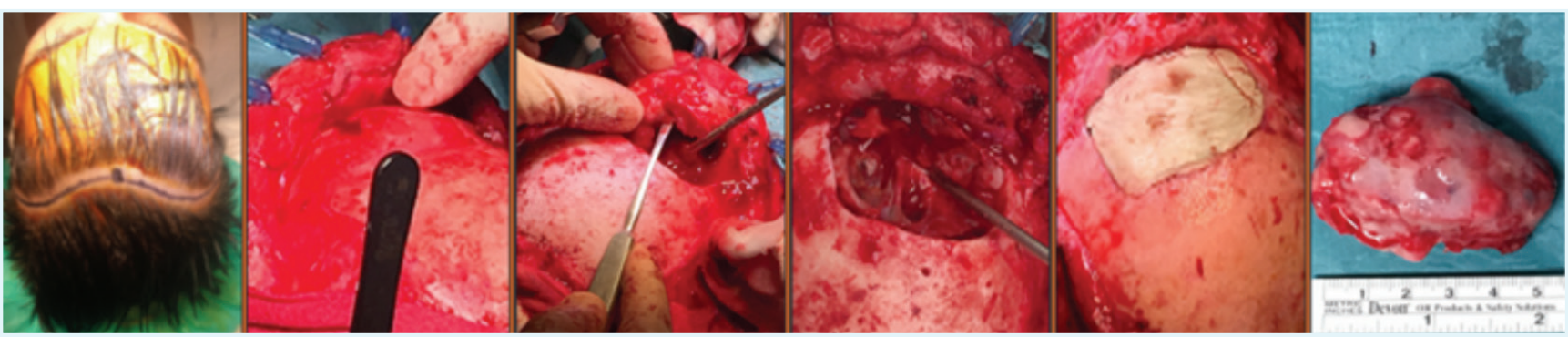

FIGURA 3. Cirurgia multidisciplinar com ORL e Neurocirurgia. Realizada exérese de lesão fibrodisplásica frontal e da fossa anterior através de craniotomia bicoronal com encerramento da dura-máter e do seio frontal com retalho de pericrânio e reconstrução do defeito ósseo por cranioplastia.

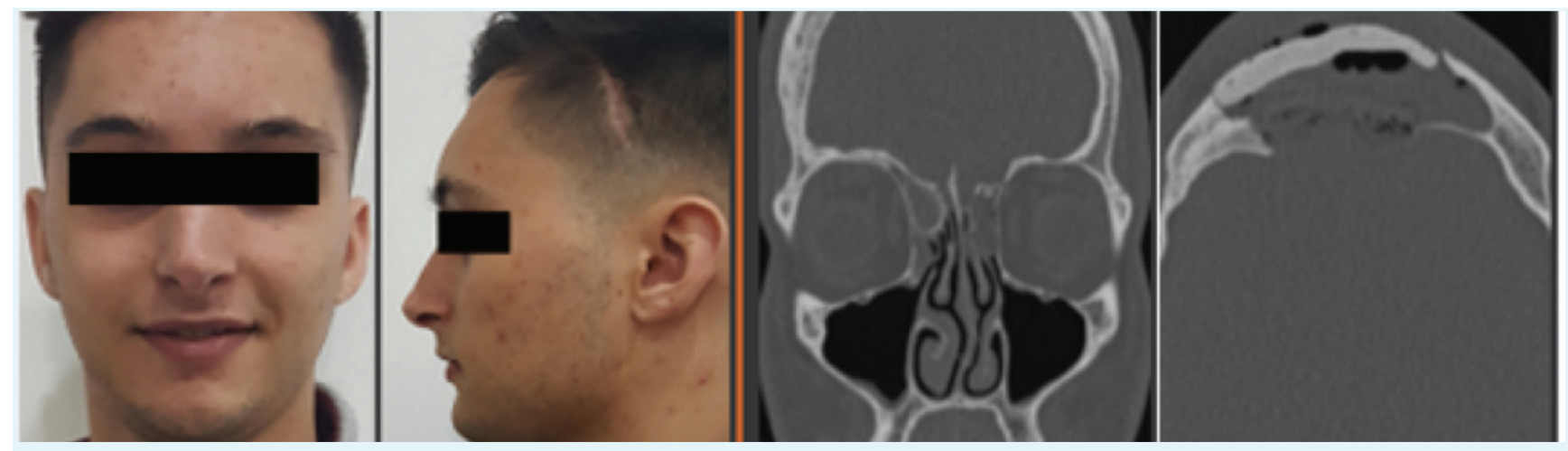

FIGURA 4. Um ano de seguimento, sem evidência clínica ou imagiológica de recidiva.
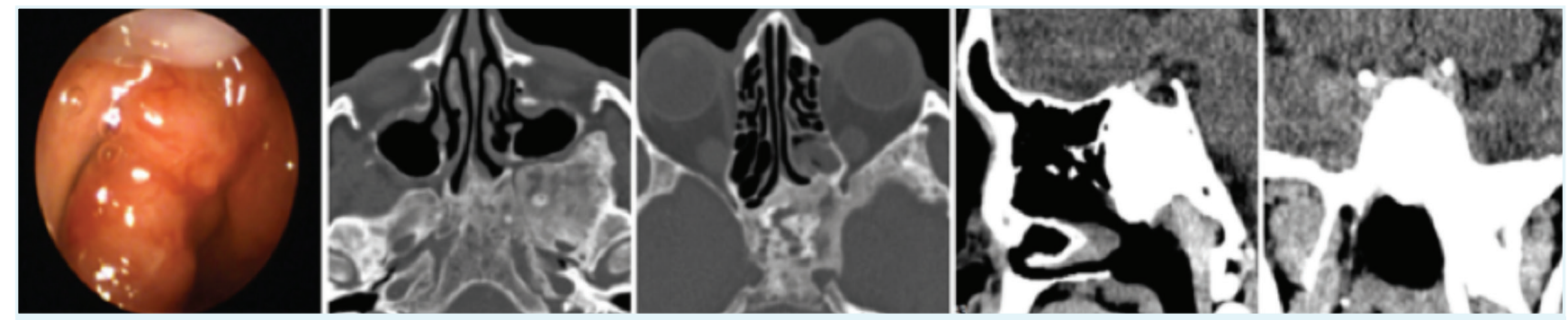

FIGURA 5. Tumefação da nasofaringe que motivou realização de TC, que revelou alterações compatíveis com FOM esfenoidal, condicionando obliteração praticamente total do seio esfenoidal, envolvimento do corpo, grande asa e apófise pterigoide, à esquerda.

endoscópica nasossinusal (se possível com auxílio de navegação) nos casos limitados e por abordagem combinada (endonasal e externa) nos casos mais extensos, seguido de obliteração do seio com material autólogo como músculo, gordura ou retalhos locais como pericrânio. ${ }^{3} \mathrm{~A}$ via e extensão da ressecção dependem da localização, envolvimento de estruturas adjacentes e proximidade a estruturas importantes como a órbita, o nervo ótico, as artérias etmoidais, estruturas do seio cavernoso, como a artéria carótida interna, o III, IV, V e VI pares cranianos, e o próprio parênquima encefálico.

No caso particular da FO com envolvimento do seio esfenoidal e base do crânio, é necessário avaliação e monitorização da acuidade visual pelo risco de envolvimento e compressão do nervo ótico. Nestes casos, a descompressão profilática do nervo ótico é advogada por alguns autores, contudo a sua realização não é consensual, uma vez que mesmo com a sua realização, 5\% a 33\% dos pacientes não melhoram a sua acuidade visual, podendo agravar ou mesmo correr o risco de amaurose. ${ }^{3}$ Os autores mais conservadores, defendem a vigilância oftalmológica periódica com determinação da acuidade visual, campimetria e exame do fundo ocular. ${ }^{3}$

O tratamento com bifosfonatos está indicado nos casos de descontrolo álgico e para controlo do crescimento da lesão. 3,7

A observação e vigilância têm indicação nos casos assintomáticos, sem evidência imagiológica de crescimento. 3,6

O prognóstico é favorável dada a transformação maligna rara, contudo o seu caráter recidivante torna o seguimento imprescindível, sobretudo nas formas de apresentação mais jovens. 


\section{CONCLUSÃO}

A FO é uma patologia rara que pode ter um amplo espetro de apresentação, comportamento e evolução. Tipicamente afeta indivíduos jovens, tem comportamento benigno e crescimento lento, podendo invadir e causar compressão de estruturas nobres da base do crânio e órbita. Estes aspetos devem ser considerados na orientação e sobretudo no planeamento cirúrgico. A cirurgia deve estabelecer um equilíbrio entre a remoção completa da lesão, dado o seu caráter recidivante, e a preservação de estruturas nobres, de forma a evitar défices funcionais. Os bifosfonatos são uma opção válida para controlo álgico e estabilização de lesões em fase estável.

A predileção craniofacial da FO enfatiza que seja um diagnóstico a considerar na prática médica do otorrinolaringologista.

CONFLITOS DE INTERESSE: Os autores declaram não ter qualquer conflito de interesse na realização do presente trabalho.

FONTES DE FINANCIAMENTO: Não houve qualquer fonte de financiamento na realização do presente trabalho.

CONFIDENCIALIDADE DOS DADOS: Os autores declaram ter seguido os protocolos da sua instituição acerca da publicação dos dados de doentes.

PROTEÇÃO DE PESSOAS E ANIMAIS: Os autores declaram que os procedimentos seguidos na elaboração do presente trabalho estão em conformidade com as normas das comissões de investigação clínica e de ética, bem como da declaração de Helsínquia e da Associação Médica Mundial.

\section{CONSENTIMENTO DO DOENTE: Obtido.}

CONFLICTS OF INTEREST: The authors declare that they have no conflicts of interest.

FINANCIAL SUPPORT: This work has not received any contribution, grant or scholarship.

CONFIDENTIALITY OF DATA: The authors declare that they have followed the protocols of their work center on the publication of data from patients.

PROTECTION OF HUMAN AND ANIMAL SUBJECTS: The authors declare that the procedures followed were in accordance with the regulations of the relevant clinical research ethics committee and with those of the Code of Ethics of the World Medical Association (Declaration of Helsinki).

PATIENT CONSENT: Obtained.

\section{REFERÊNCIAS}

1. Couturier A, Aumaître O, Gilain L, Jean B, Mom T, André M. Craniofacial fibrous dysplasia: A 10-case series. Eur Ann Otorhinolaryngol Head Neck Dis. 2017;134:229-35.

2. Tabareau-Delalande F, Collin C, Gomez-Brouchet A, Decouvelaere AV, Bouvier C, Larousserie F, et al. Diagnostic value of investigating GNAS mutations in fibro-osseous lesions: a retrospective study of 91 cases of fibrous dysplasia and 40 other fibro-osseous lesions. Mod Pathol. 2013;26:911-21.

3. Lee JS, FitzGibbon EJ, Chen YR, Kim HJ, Lustig LR, Akintoye $\mathrm{SO}$, et al. Clinical guidelines for the management of craniofacial fibrous dysplasia. Orphanet J Rare Dis. 2012;7 Suppl 1:S2.

4. Bousson V, Rey-Jouvin C, Laredo JD, Le Merrer M, Martin-Duverneuil N, Feydy A, et al. Fibrous dysplasia and McCune-Albright syndrome: imaging for positive and differential diagnoses, prognosis, and follow-up guidelines. Eur J Radiol 2014;83:1828-42.

5. Eller R, Sillers M. Common fibro-osseous lesions of the paranasal sinuses. Otolaryngol Clin North Am. 2006; 39:585-600.

6. Amit M, Collins MT, FitzGibbon EJ, Butman JA, Fliss DM, Gil Z. Surgery versus watchful waiting in patients with craniofacial fibrous dysplasia-a meta-analysis. PloS One. 2011; 6:e25179.

7. Chao K, Katznelson L. Use of high-dose oral bisphosphonate therapy for symptomatic fibrous dysplasia of the skull. J Neurosurg. 2008;109:889-92. 